\title{
Analytical Solution for a Problem of Directional Solidification in a Ternary System
}

\author{
D.V. Alexandrov and A.A. Ivanov \\ Department of Mathematical Physics, Urals State University \\ Ekaterinburg 620083, Russian Federation
}

\begin{abstract}
This paper is devoted to new analytic results relating to the nonstationary dynamics of directional solidification of ternary solutions with two moving layers of the phase transition lying between solid and liquid phases. Explicit analytical solutions of the problem under consideration are constructed on the basis of laboratory experiments carried out by Aitta, Huppert and Worster.
\end{abstract}

PACS numbers: 44.05.+e, 81.30.Fb

\section{Introduction}

The seemingly trivial moving boundary problem of the crystallization of a solid phase from a cooled wall comes under the rubric of the so-called Stefan problems describing a wide range of physical processes. Their rich nonlinear behavior has attracted substantial scientific interest and their ubiquity in fields ranging from metallurgy to geophysics stimulates developing new mathematical approaches (including phase-field models) (see, among others $[1-8])$. Solidification of single-component and binary solutions within the framework of the classical frontal approach as well as of the mushy layer scenario has intensively been studied by many authors for the last few years. Some many natural and industrial processes frequently met in practice cannot be explained in terms of single-component or binary systems but can, at least partially, be understood in terms of ternary systems. The major features of the dynamics of ternary systems can be studied using laboratory system of two salts dissolved in water. The mathematical model under consideration and its analytical solution are based on laboratory experiments [9] where a ternary solution was cooled from below and all convection was suppressed because the buoyancy of the fluid released on crystallization always increased. The present study is concerned with new analytic results on the nonlinear dynamics of solidification of a three-component alloy with two mushy layers on the basis of experimental data on crystallization of the ternary alloy $\mathrm{H}_{2} \mathrm{O}-\mathrm{KNO}_{3}-\mathrm{NaNO}_{3}$.

\section{The moving boundary problem and its analytical solution}

Let us consider a unidirectional solidification process generated by the external cooling of the solid boundary $x=0$. Figure 1 demonstrates a scheme of the solidification process. A semi-infinite zone $x>0$ is filled with liquid $x>p(t)$ with initially uniform compositions
$C_{\infty}, B_{\infty}$ and temperature $T_{\infty}$, solid $0<x<e(t)$ and two mushy layers, primary $c(t)<x<p(t)$ and cotectic $e(t)<x<c(t)$. The solid boundary $x=0$ is maintained at the atmospheric temperature $T_{0}<T_{\infty}$. Two components with impurity concentrations $C$ and $B$ are dissolved in solvent $A$ so that the primary mushy layer characterizes the phase transition of solvent $A$ whereas the cotectic mushy layer describes freezing of components $A$ and $B$. The eutectic solid phase is made up of components $A, B$, and $C$ in the solid state. Keeping in mind the phase diagram of the ternary alloy $\mathrm{H}_{2} \mathrm{O}-\mathrm{KNO}_{3}-\mathrm{NaNO}_{3}$ $[9,10]$ and experimental conditions [9], we arrive at the following model of heat and mass transfer equations and boundary conditions imposed at the moving boundaries solid phase-cotectic mushy layer, cotectic mushy layerprimary mushy layer and primary mushy layer-liquid phase. For the sake of simplicity, equations and their approximate analytical solutions will be discussed further separately in each of the regions.

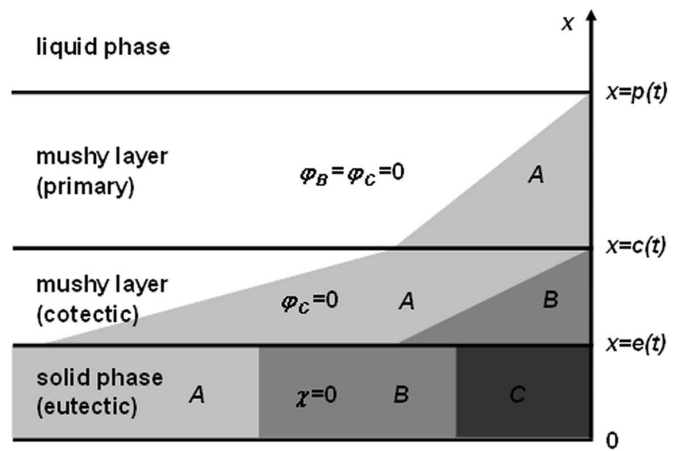

Fig. 1. A schematic diagram of directional solidification of a ternary solution $(x-$ spatial coordinate, $p(t)$, $c(t)$ and $e(t)$ - moving boundaries of the phase transition). Shaded zones demonstrate solid fractions of components $A, B$ and $C$ ( $B$ and $C$ are dissolved in $A$, $A+B+C=1)$. 
The heat-conduction and diffusion equations in the primary mushy layer $\left(\varphi_{B}=\varphi_{C}=0\right)$ can be written in the form $[1,10]$ :

$$
\begin{gathered}
\rho_{p} c_{p} \frac{\partial T}{\partial t}=\frac{\partial}{\partial x}\left[k_{p}(\chi) \frac{\partial T}{\partial x}\right]+L_{V} \frac{\partial \varphi_{A}}{\partial t}, \\
c(t)<x<p(t), \\
\chi \frac{\partial B}{\partial t}=D_{B} \frac{\partial}{\partial x}\left(\chi \frac{\partial B}{\partial x}\right)+B \frac{\partial \varphi_{A}}{\partial t}, \\
c(t)<x<p(t), \\
\chi \frac{\partial C}{\partial t}=D_{C} \frac{\partial}{\partial x}\left(\chi \frac{\partial C}{\partial x}\right)+C \frac{\partial \varphi_{A}}{\partial t}, \\
c(t)<x<p(t),
\end{gathered}
$$

where $T$ is the temperature in the primary mushy layer, $\rho_{p}$ is the density, $c_{p}$ is the heat capacity, $L_{V}$ is the latent heat, $D$ is the diffusion coefficient, $\varphi$ is the solid fraction, $\chi=1-\varphi_{A}$ is the liquid fraction and $t$ is the solidification time (subscripts $A, B$, and $C$ designate respective components) The heat conductivity $k_{p}$ depends on $\chi$ and constant thermal conductivities of the solid, $k_{\mathrm{S}}$ and liquid $k_{\mathrm{L}}[10]: k(\chi)=k_{\mathrm{L}} \chi+k_{\mathrm{S}}(1-\chi)$.

The phase transition temperature $T$ and compositions $B$ and $C$ in the primary mushy layer are related by means of the liquidus surface equation [10]:

$$
T=T_{\mathrm{M}}+m_{B} B+m_{C} C, \quad c(t)<x<p(t),
$$

where $T_{\mathrm{M}}$ is the phase transition temperature of the pure material, $m_{B}$ and $m_{C}$ are the constant liquidus slopes.

Taking into account that relaxation times of the solute concentration fields are several orders of magnitude higher than the thermal relaxation time, the temperature field in the primary mushy layer can be written as a linear function of coordinate $x$ (see also experimental data $[9])$ :

$$
T(t, z)=T_{1}(t)+T_{2}(t) x, \quad c(t)<x<p(t) .
$$

Omitting the left-hand side of Eq. (1) due to the fact that the relaxation times of impurity concentration fields exceed greatly the temperature relaxation time, we obtain from (1) and (5) the following dependence between spatial and time derivatives:

$$
T_{2}(t) \frac{\partial \varphi_{A}}{\partial x}=\frac{L_{V}}{k_{\mathrm{L}}-k_{\mathrm{S}}} \frac{\partial \varphi_{A}}{\partial t}, \quad c(t)<x<p(t) .
$$

Now, substitution of (5) and (6) into (2) and (3) gives the desired solution in the form

$$
\begin{aligned}
& B(t, x)=\frac{b_{2} T_{D} \varphi_{A}+B_{p b}}{1-\varphi_{A}}, \\
& C(t, x)=\frac{c_{2} T_{D} \varphi_{A}+C_{p b}}{1-\varphi_{A}}, \\
& T_{D}=\frac{L_{V} D_{B}}{k_{\mathrm{S}}-k_{\mathrm{L}}}, \\
& c(t)<x<p(t) .
\end{aligned}
$$

Here, for the sake of simplicity we consider that $D_{B}=D_{C}\left(B_{p b}\right.$ and $C_{p b}$ stand for the impurity concentrations at $x=p(t)$ or $\left.\varphi_{A}=0\right)$. Also, we assume that the compositions $B$ and $C$ are linear functions of $x$ with corresponding derivatives $B_{2}(t)=b_{2} T_{2}(t)$ and
$C_{2}(t)=c_{2} T_{2}(t)$ proportional to the temperature gradient, $B(t, x)=B_{1}(t)+B_{2}(t) x$ and $C(t, x)=C_{1}(t)+$ $C_{2}(t) x$. This representation of the compositions $B$ and $C$ follows from the linear form of Eq. (4) by analogy with binary mixtures.

Combining expressions (4), (5) and (7), we find the solid fraction in the primary mushy layer

$$
\begin{gathered}
\varphi_{A}(t, x)=\frac{T_{1}+T_{2} x-T_{\mathrm{M}}-m_{B} B_{p b}-m_{C} C_{p b}}{T_{1}+T_{2} x-T_{\mathrm{M}}+T_{D}}, \\
c(t)<x<p(t),
\end{gathered}
$$

where we used that $m_{B} b_{2}+m_{C} c_{2}=1$.

Combining expressions (7) and (8), we come to

$$
b_{2}=\frac{B_{p b}}{m_{B} B_{p b}+m_{C} C_{p b}}, \quad c_{2}=\frac{C_{p b}}{m_{B} B_{p b}+m_{C} C_{p b}} .
$$

Substituting these constants and the solid fraction (8) into (7), we finally arrive at the compositions in the primary mushy layer

$$
\begin{gathered}
B(t, x)=\frac{B_{p b}\left(T_{1}+T_{2} x-T_{\mathrm{M}}\right)}{m_{B} B_{p b}+m_{C} C_{p b}}, \\
C(t, x)=\frac{C_{p b}\left(T_{1}+T_{2} x-T_{\mathrm{M}}\right)}{m_{B} B_{p b}+m_{C} C_{p b}}, \\
c(t)<x<p(t) .
\end{gathered}
$$

We call attention to the special case of the Scheil form of diffusion Eqs. (2) and (3) when the first terms in the right-hand sides of (2) and (3) are omitted. This representation in the absence of diffusion leads to the same distributions (9). The reason is that the diffusion field in the mushy layer is frozen and the mass transport is caused by the impurity displacement into the liquid phase.

Further, let us assume that the impurity displacement rate and the phase transition rate of boundary $x=p(t)$ coincide very closely due to the fact that the diffusion profile at $x>p(t)$ is nearly constant [9]. Therefore, mass balances at the interface primary mushy layerliquid phase can be written as

$$
\begin{aligned}
& \left(B_{\infty}-B_{p b}\right) \frac{\mathrm{d} p}{\mathrm{~d} t}=D_{B} \frac{\partial B}{\partial x}, \\
& \left(C_{\infty}-C_{p b}\right) \frac{\mathrm{d} p}{\mathrm{~d} t}=D_{C} \frac{\partial C}{\partial x}, \quad x=p(t) .
\end{aligned}
$$

Substituting (9) into (10), we get a relation connecting the interface concentrations $\left(D_{B}=D_{C}\right)$ :

$$
C_{p b}=\frac{C_{\infty}}{B_{\infty}} B_{p b} .
$$

Combining (11) and first expressions in (9) and (10), we have

$$
\begin{aligned}
& \left(B_{\infty}-B_{p b}\right) R \frac{\mathrm{d} p}{\mathrm{~d} t}=D_{B} B_{\infty} T_{2}, \\
& R=m_{B} B_{\infty}+m_{C} C_{\infty} .
\end{aligned}
$$

Equating temperature (4) and the interface temperature at $x=p(t)$ and taking into account (11), we obtain the interface concentration $B_{p b}$ in the form

$$
B_{p b}=\left(T_{p b}-T_{M}\right) B_{\infty} / R \text {. }
$$

Now, substituting $B_{p b}$ from (13) into (12) and taking into account (5) at $x=p(t)$, we get the temperature 
coefficients in the primary mushy layer

$$
\begin{aligned}
& T_{1}(t)=T_{p b}-p(t) T_{2}(t), \\
& T_{2}(t)=\frac{R+T_{\mathrm{M}}-T_{p b}}{D_{B}} \frac{\mathrm{d} p}{\mathrm{~d} t} .
\end{aligned}
$$

Laboratory experiments [9] show that the phase transition temperature $T_{p b}$ is very nearly constant. On this basis, let us represent the temperature field in the liquid phase as

$$
\begin{aligned}
& T(t, x)=T_{\infty}+\left(T_{p b}-T_{\infty}\right) \frac{\operatorname{erfc}(x / 2 \sqrt{\kappa t})}{\operatorname{erfc}(p(t) / 2 \sqrt{\kappa t})}, \\
& \quad x>p(t),
\end{aligned}
$$

where $\kappa$ is the thermal diffusivity coefficient in the liquid. From the mathematical point of view distribution (14) is valid only if $T_{p b}$ is constant. Physically, we use this temperature profile known from the theory of self-similar solidification as approximation for small time oscillations of the interface temperature. One further comment should be made. Also, distribution (14) is valid if $p(t) \propto \sqrt{t}$ (the latter follows from our subsequent theory).

Equating temperature derivatives at $x=p(t)$ and keeping in mind expressions (5) and (14), we come to a relation connecting two unknowns $p(t)$ and $T_{p b}$ :

$$
\begin{aligned}
& \frac{\left(T_{\infty}-T_{p b}\right) D_{B} \exp \left(-p^{2}(t) / 4 \kappa t\right)}{\sqrt{\pi \kappa t}\left(R+T_{\mathrm{M}}-T_{p b}\right)}= \\
& \operatorname{erfc}(p(t) / 2 \sqrt{\kappa t}) \frac{\mathrm{d} p}{\mathrm{~d} t},
\end{aligned}
$$

where temperature $T_{p b}$ should be constant.

The heat and mass transfer in the cotectic mushy layer $\left(\varphi_{C}=0, \chi=1-\varphi_{A}-\varphi_{B}\right)$ is described by the following set of equations $[10]$ :

$$
\begin{aligned}
\rho_{c} c_{c} \frac{\partial T}{\partial t}= & \frac{\partial}{\partial x}\left[k_{c}(\chi) \frac{\partial T}{\partial x}\right]+L_{V} \frac{\partial\left(\varphi_{A}+\varphi_{B}\right)}{\partial t}, \\
& e(t)<x<c(t), \\
\chi \frac{\partial B}{\partial t}= & D_{B} \frac{\partial}{\partial x}\left(\chi \frac{\partial B}{\partial x}\right)+B \frac{\partial\left(\varphi_{A}+\varphi_{B}\right)}{\partial t}-\frac{\partial \varphi_{B}}{\partial t}, \\
& e(t)<x<c(t), \\
\chi \frac{\partial C}{\partial t}=D_{C} \frac{\partial}{\partial x}\left(\chi \frac{\partial C}{\partial x}\right)+C \frac{\partial\left(\varphi_{A}+\varphi_{B}\right)}{\partial t}, & e(t)<x<c(t),
\end{aligned}
$$

where $T$ is the temperature in the cotectic mushy layer, $\rho_{c}$ is the density, $c_{c}$ is the heat capacity.

Again, keeping in mind that a relaxation time of the temperature field in the cotectic region is greatly smaller than relaxation times of the diffusion fields, we approximate the temperature profile as linear function of $x$ (we use (19) instead of (16)):

$$
T(t, x)=T_{3}(t)+T_{4}(t) x, \quad e(t)<x<c(t) .
$$

Let us write down two equations connecting the temperature and impurity concentrations in the secondary mushy layer. Taking into consideration Eq. (19), we have [10]:

$$
\begin{aligned}
B(t, x)= & -\frac{1}{m_{B}^{c}}\left(T_{3}+T_{4} x-T_{E}\right)+B_{E}, \\
& e(t)<x<c(t), \\
C(t, x)= & -\frac{1}{m_{C}^{c}}\left(T_{3}+T_{4} x-T_{E}^{A B}\right), \\
& e(t)<x<c(t),
\end{aligned}
$$

where constants $m_{B}^{c}, m_{C}^{c}, T_{E}, B_{E}$ and $T_{E}^{A B}$ are known from the phase diagram [10].

In order to integrate the mass transfer Eqs. (17) and (18), we express the temperature derivative with respect to time in terms of its spatial analog by means of Eq. (16). As before, omitting the left-hand side of Eq. (16) and substituting the linear temperature profile from Eq. (19), we arrive at a relation, similar to (6):

$$
\begin{gathered}
T_{4} \frac{\partial\left(1-\varphi_{A}-\varphi_{B}\right)}{\partial x}=\frac{L_{V}}{k_{\mathrm{L}}-k_{\mathrm{S}}} \frac{\partial\left(1-\varphi_{A}-\varphi_{B}\right)}{\partial t}, \\
e(t)<x<c(t) .
\end{gathered}
$$

Now, combining (17), (18), (20) and (21), we find the compositions $B$ and $C$ in the cotectic mushy layer

$$
\begin{aligned}
B(t, x)= & \frac{\left(\chi-\chi_{\mathrm{S}}^{-}\right) T_{D} / m_{B}^{c}+B_{\mathrm{S} b} \chi_{\mathrm{S}}^{-}+\varphi_{B \mathrm{~S}}^{-}-\varphi_{B}}{\chi}, \\
& e(t)<x<c(t), \\
C(t, x)= & \frac{\left(\chi-\chi_{\mathrm{S}}^{-}\right) T_{D} / m_{C}^{c}+C_{\mathrm{S} b} \chi_{\mathrm{S}}^{-}}{\chi} \\
& e(t)<x<c(t),
\end{aligned}
$$

where $\chi_{\mathrm{S}}^{-}=1-\varphi_{A \mathrm{~S}}^{-}-\varphi_{B \mathrm{~S}}^{-}, \chi(t, x)=1-\varphi_{A}(t, x)-$ $\varphi_{B}(t, x), B_{\mathrm{S} b}$ and $C_{\mathrm{S} b}$ are the solute concentrations at $x=c(t), \varphi_{A \mathrm{~S}}^{-}$and $\varphi_{B \mathrm{~S}}^{-}$are the boundary values of $\varphi_{A}$ and $\varphi_{B}$ at the left side of boundary $x=c(t)$.

Experimental data [9] show that the temperature field in both the mushy layers can be approximated by a single linear function of $x$, that is $T_{1}=T_{3}$ and $T_{2}=T_{4}$.

Equating expressions (9) and (20) (to do this, we substitute $B$ or $C$ from (20)), we obtain the phase transition boundary $c(t)$ as a composed function of time

$$
\begin{aligned}
& c(t)=p(t) \\
& \quad+\frac{T_{E}^{A B} R+m_{C}^{c} C_{\infty} T_{\mathrm{M}}-\left(m_{C}^{c} C_{\infty}+R\right) T_{p b}}{\left(m_{C}^{c} C_{\infty}+R\right) T_{2}} .
\end{aligned}
$$

Combining expressions (8) and (24) at $x=c(t)$, we have the solid fraction at the right side of this boundary

$$
\begin{aligned}
& \varphi_{A \mathrm{~S}}^{+}=1 \\
& \quad-\frac{\left(m_{C}^{c} C_{\infty}+R\right)\left(R B_{p b}+T_{D} B_{\infty}\right)}{B_{\infty}\left[\left(T_{E}^{A B}+T_{D}-T_{\mathrm{M}}\right) R+m_{C}^{c} T_{D} C_{\infty}\right]} .
\end{aligned}
$$

Equating the left-hand sides of (20), (22) and (23) and omitting mathematical manipulations, we get the solid fractions in the cotectic mushy layer

$$
\begin{gathered}
\varphi_{B}(t, x)=\varphi_{B \mathrm{~S}}^{-}+\left(B_{\mathrm{S} b}-T_{D} / m_{B}^{c}\right) \chi_{\mathrm{S}}^{-}+ \\
\frac{\left(T_{D}-m_{C}^{c} C_{\mathrm{S} b}\right)\left(T_{1}+T_{2} x-T_{E}+T_{D}-m_{B}^{c} B_{E}\right)}{m_{B}^{c}\left(T_{1}+T_{2} x+T_{D}-T_{E}^{A B}\right)} \chi_{\mathrm{S}}^{-},
\end{gathered}
$$




$$
\begin{aligned}
& \varphi_{A}(t, x)=1-\varphi_{B}(t, x) \\
& -\frac{T_{D}-m_{C}^{c} C_{\mathrm{S} b}}{T_{1}+T_{2} x+T_{D}-T_{E}^{A B}} \chi_{\mathrm{S}}^{-} .
\end{aligned}
$$

The solid fractions $\varphi_{A S}^{-}$and $\varphi_{B S}^{-}$at the left side of boundary $x=c(t)$ are determined by the mass balance boundary conditions [10]. Taking into consideration formulae (9) and (20), we rewrite these conditions in the form $\left(D_{B}=D_{C}, T_{2}=T_{4}\right)$

$$
\begin{gathered}
D_{B} T_{2}\left[\left(1-\varphi_{A \mathrm{~S}}^{-}-\varphi_{B \mathrm{~S}}^{-}\right) R+\left(1-\varphi_{A \mathrm{~S}}^{+}\right) m_{B}^{c} B_{\infty}\right]= \\
m_{B}^{c} R\left[\varphi_{B \mathrm{~S}}^{-}+\left(\varphi_{A \mathrm{~S}}^{+}-\varphi_{A \mathrm{~S}}^{-}-\varphi_{B \mathrm{~S}}^{-}\right) B_{\mathrm{S} b}\right] \frac{\mathrm{d} c}{\mathrm{~d} t}, \\
D_{B} T_{2}\left[\left(1-\varphi_{A \mathrm{~S}}^{-}-\varphi_{B \mathrm{~S}}^{-}\right) R+\left(1-\varphi_{A \mathrm{~S}}^{+}\right) m_{C}^{c} C_{\infty}\right]= \\
m_{C}^{c} R C_{\mathrm{S} b}\left(\varphi_{A \mathrm{~S}}^{+}-\varphi_{A \mathrm{~S}}^{-}-\varphi_{B \mathrm{~S}}^{-}\right) \frac{\mathrm{d} c}{\mathrm{~d} t} .
\end{gathered}
$$

Taking into account that the concentration field is continuous at $x=c(t)$, we have $C_{\infty} B_{\mathrm{S} b}=B_{\infty} C_{\mathrm{S} b}$.

Keeping in mind that the temperature field in the solid region, $0<x<e(t)$, coincides with the temperature field in two mushy layers, we can express this function in the form

$$
\begin{gathered}
T(t, x)=T_{0}-\frac{T_{0}-T_{E}}{e(t)} x, \quad 0<x<e(t), \\
T_{1}(t)+T_{2}(t) e(t)=T_{E},
\end{gathered}
$$

where $T_{0}$ is the temperature maintained at the solid wall $x=0$; whence it follows that

$$
e(t)=\frac{T_{E}-T_{1}}{T_{2}}=p(t)+\frac{T_{E}-T_{p b}}{T_{2}} .
$$

Keeping in mind that $T_{2} e(t)=T_{E}-T_{0}$, we find the solid phase--cotectic mushy layer interface coordinate in the form

$$
e(t)=\frac{T_{0}-T_{E}}{T_{0}-T_{p b}} p(t)
$$

Eliminating $e(t)$ from expressions (30) and (31), we arrive at the following law of motion of the primary mushy layer-liquid phase boundary:

$$
p(t)=\sqrt{p(0)+\sigma D_{B} t}, \quad \sigma=\frac{2\left(T_{p b}-T_{0}\right)}{R+T_{\mathrm{M}}-T_{p b}} .
$$

Now, substitution of $p(t)$ from (32) into (15) confirms our hypothesis about constant temperature $T_{p b}$. As a result, we come to the following transcendental equation for the determination of $T_{p b}$ :

$$
\frac{T_{p b}-T_{\infty}}{T_{0}-T_{p b}} \sqrt{\frac{\sigma D_{B}}{\pi \kappa}}=\operatorname{erfc}\left(\sqrt{\frac{\sigma D_{B}}{4 \kappa}}\right) \exp \left(\frac{\sigma D_{B}}{4 \kappa}\right) .
$$

Now we can conclude that the process under consideration is self-similar because three boundaries move as square root functions of time (see expressions (24), (31) and (32)) and boundary temperatures are invariable.

Keeping in mind that $T_{p b}-T_{0}=T_{2} p(t)$ and $\mathrm{d} p / \mathrm{d} t=$ $\sigma D_{B} / 2 p(t)$, we represent the rate of boundary $c(t)$ in the form

$$
\begin{aligned}
\frac{\mathrm{d} c}{\mathrm{~d} t} & =\frac{D_{B} W}{p(t)}, \\
W & =\frac{\left(T_{\mathrm{M}}-T_{0}\right) m_{C}^{c} C_{\infty}+\left(T_{E}^{A B}-T_{0}\right) R}{\left(m_{C}^{c} C_{\infty}+R\right)\left(R+T_{\mathrm{M}}-T_{p b}\right)} .
\end{aligned}
$$

Dividing (28) by (29) and taking into account (33) we find the solid fractions $\varphi_{A \mathrm{~S}}^{-}$and $\varphi_{B \mathrm{~S}}^{-}$

$$
\varphi_{A \mathrm{~S}}^{-}=s-\varphi_{B \mathrm{~S}}^{-} \quad s=\frac{C_{\infty}\left(T_{p b}-T_{0}\right)\left(1-\varphi_{A \mathrm{~S}}^{+}\right)+R\left(T_{p b}-T_{0}\right) / m_{C}^{c}-W R C_{\mathrm{S} b} \varphi_{A \mathrm{~S}}^{+}}{R\left(T_{p b}-T_{0}\right) / m_{C}^{c}-W R C_{\mathrm{S} b}},
$$

$$
\begin{aligned}
& \varphi_{B \mathrm{~S}}^{-}=C_{\mathrm{S} b}\left(\varphi_{A \mathrm{~S}}^{+}-s\right) \frac{(1-s) R / m_{B}^{c}+\left(1-\varphi_{A \mathrm{~S}}^{+}\right) B_{\infty}}{(1-s) R / m_{C}^{c}+\left(1-\varphi_{A \mathrm{~S}}^{+}\right) C_{\infty}} \\
& \quad+B_{\mathrm{S} b}\left(s-\varphi_{A \mathrm{~S}}^{+}\right) .
\end{aligned}
$$

The solid fractions $\varphi_{A e}^{+}$and $\varphi_{B e}^{+}$on the right side of boundary $e(t)$ can be found from expressions (26) and (27) by substitution of $x=e(t)$ whereas the boundary values $\varphi_{B e}^{-}$and $\varphi_{C e}^{-}$on the left side of this phase transition interface can be found from the mass balance condition imposed at $x=e(t)$ [10]. Omitting mathematical details, we have

$$
\begin{aligned}
& \varphi_{B e}^{-}=\varphi_{B e}^{+}+B_{E} \Theta-\frac{D_{B} \Theta T_{2}}{m_{B}^{c} \mathrm{~d} e / \mathrm{d} t} \\
& \varphi_{C e}^{-}=C_{E} \Theta-\frac{D_{C} \Theta T_{2}}{m_{C}^{c} \mathrm{~d} e / \mathrm{d} t}, \quad \Theta=1-\varphi_{A e}^{+}-\varphi_{B e}^{+},
\end{aligned}
$$

where compositions $B_{E}$ and $C_{E}$ are known from the phase diagram and $\varphi_{A e}^{-}=1-\varphi_{B e}^{-}-\varphi_{C e}^{-}$.

\section{Results and discussions}

The temperature profile in the solidification region is plotted in Fig. 2. It is easily seen that the temperature field is linear in the solid phase and two mushy layers whereas its behavior differs from a linear dependence in the liquid region. The reason is that the growing solid phase displaces dissolved impurities into the liquid matrix and, as a result, the solute concentration in the vicinity of the ingot mould $(x \rightarrow \infty)$ increases with time. The latter leads to a decrease in the phase transition temperature. This fact combined with a constant temperature $T_{\infty}$ explains a temperature departure from linearity. As may be seen from Fig. 3, the solid fractions in two mushy layers decrease away from the boundary solid 


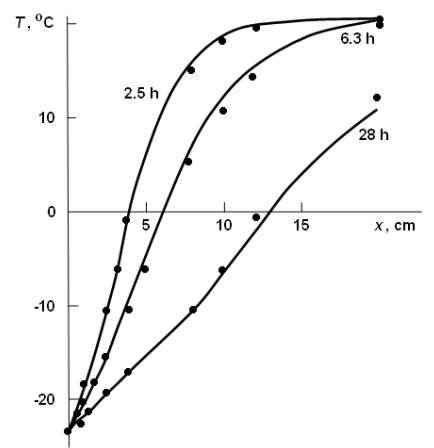

Fig. 2. The temperature distribution in the solidification region in accordance with the theory under consideration and experimental data [7] (black circles, experiment 7) for different time points (numbers at the curves). Physical parameters are given in Refs. [7] and $[8]$.

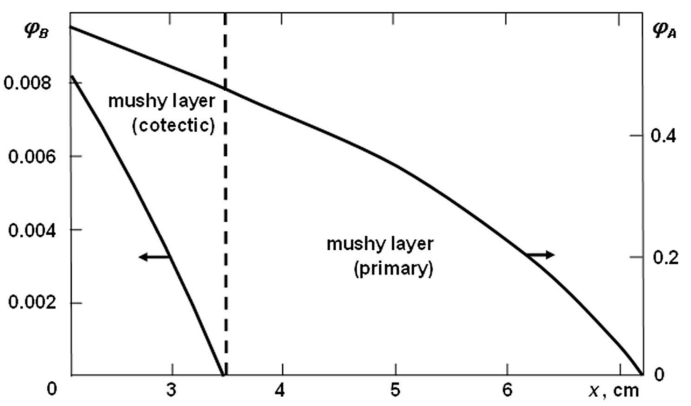

Fig. 3. The solid fractions in cotectic and primary mushy layers at the point of time $t=10^{5} \mathrm{~s}$ in accordance with the theory under consideration.

phase-cotectic mushy layer. Such a behavior corresponds to the classical solidification theory of binary mixtures (see, among others [1]). As would be expected, the liquid composition $C$ decreases with $x$ due to the effect of impurity displacement by the growing crystal (Fig. 4). Contrary to this classical behavior, the liquid composition $B$ increases (decreases) with $x$ in the cotectic (primary) layer. 3D plot in Fig. 5 shows a dynamic behavior of the liquidcomposition $B$. The liquid composition $B$

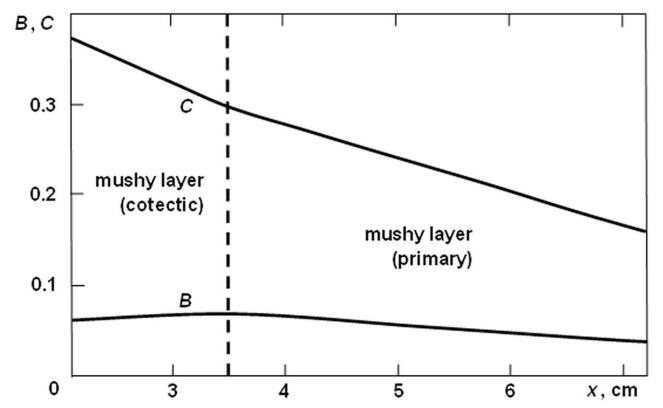

Fig. 4. The impurity concentrations of components $B$ and $C$ at the point of time $t=10^{5} \mathrm{~s}$ in accordance with the theory under consideration. attains its maximum at the moving phase transition interface cotectic mushy layer-primary mushy layer (such a behavior was observed in experiments [9]) From the mathematical point of view, this is due to the fact that coefficients $m_{B}, m_{C}$ and $m_{B}^{c}$ are negative [10] (distributions (9) and (20)). Physically, the reason is that the liquid composition $B$ undergoes a phase transition in the cotectic mushy layer resulting in a decrease in composition $B$ in the vicinity of the phase transition interface $x=e(t)$.

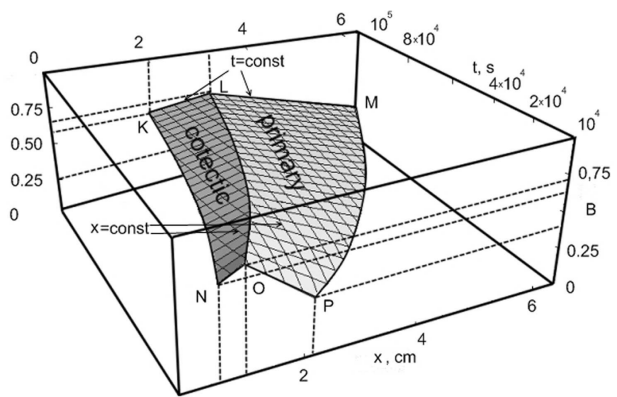

Fig. 5. 3D behavior of the liquid composition $B$ ( $B$ corresponds to $\mathrm{KNO}_{3}$ ). Points $\mathrm{N}, \mathrm{O}, \mathrm{P}$ and $\mathrm{K}, \mathrm{L}, \mathrm{M}$ lie in the time planes $t=10^{4} \mathrm{~s}$ and $t=10^{5} \mathrm{~s}$, respectively.

\section{Acknowledgments}

This work was made possible due to the financial support of grants Nos. 08-01-00298, 09-08-00844, 07-0396069 Ural, 07-01-96091 Ural (Russian Foundation for Basic Research), MD-4563.2008.2. (President Grant) and 2.1.1/2571 (Ministry of Education).

\section{References}

[1] Yu.A. Buyevich, D.V. Alexandrov, V.V. Mansurov, Macrokinetics of Crystallization, Begell House, New York 2001.

[2] R.N. Hills, D.E. Loper, P.H. Roberts, Quantum J. Appl. Math. 36, 505 (1983).

[3] D.V. Alexandrov, I.G. Nizovtseva, Dokl. Earth Sci. 419, 359 (2008).

[4] D.L. Aseev, D.V. Alexandrov, Acta Mater. 54, 2401 (2006).

[5] P.K. Galenko, O. Fanke, J. Wang, D.M. Herlach, Mater. Sci. Eng. A 375-377, 488 (2004).

[6] D.V. Alexandrov, A.P. Malygin, I.V. Alexandrova, Ann. Glaciol. 44, 118 (2006).

[7] I. Steinbach, F. Pezzolla, B. Nestler, M. Seeßelberg, R. Prieler, G.J. Schmitz, J.L.L. Rezende, Physica D 94, 135 (1996).

[8] J. Eiken, B. Böttger, I. Steinbach, Phys. Rev. E 73, 06122 (2006).

[9] A. Aitta, H.E. Huppert, M.G. Worster, J. Fluid Mech. 432, 201 (2001).

[10] D.M. Anderson, J. Fluid Mech. 483, 165 (2003). 